\title{
Preoperative chemoradiation may be more effective for esophageal squamous cell carcinoma compared with adenocarcinoma: results from 15 randomized controlled trials of 2,250 patients
}

\author{
Wen-Juan Ma ${ }^{1,2 \#}$, Qiu-Ning Zhang ${ }^{3 \#}$, Shu-Zhen Shi ${ }^{1,2}$, Xiao-Ying Zhang ${ }^{1,2}$, Ye Zhao ${ }^{1,2}$, Jin-Hui Tian ${ }^{1,2}$, \\ Xiao-Hu Wang ${ }^{3}$ \\ ${ }^{1}$ Evidence-Based Medicine Center, the School of Basic Medical Science, Lanzhou University, Lanzhou 730000, China; ${ }^{2}$ The Key Laboratory of \\ Evidence-based Medicine and Knowledge Translation of Gansu Province, Lanzhou 730000, China; ${ }^{3}$ The Oncologic Hospital of Gansu Province, \\ Lanzhou 730000, China \\ Contributions: (I) Conception and design: JH Tian, XH Wang; (II) Administrative support: XH Wang, JH Tian; (III) Collection and assembly of data: \\ WJ Ma, QN Zhang; (IV) Data analysis and interpretation: WJ Ma, QN Zhang, SZ Shi, XY Zhang, Y Zhao; (V) Manuscript writing: All authors; (VI) \\ Final approval of manuscript: All authors. \\ \#These authors contributed equally to this work as co-first authors. \\ Correspondence to: Xiao-Hu Wang. The Oncologic Hospital of Gansu Province, Lanzhou 730000, China. Email: xhwanggansu@163.com; Jin-Hui \\ Tian. Evidence-Based Medicine Center, the School of Basic Medical Science, Lanzhou University, Lanzhou 730000, China. Email: tjh996@163.com.
}

Background: Although preoperative chemoradiation (CRT) is considered a standard treatment for patients with esophageal cancer, the results obtained from a variety studies concerning this treatment type still do not completely concur. The purpose of this study is to evaluate the efficacy of preoperative CRT on different histological types in the treatment of esophageal cancer, and to clarify the benefits of preoperative CRT for esophageal cancer.

Methods: PubMed, EMBASE, Web of science and Cochrane library were searched for eligible studies published up to December 2017. Three primary out-comes were analyzed. Pooled hazard ratio (HR) and $95 \%$ confidence intervals ( $95 \% \mathrm{CI}$ ) were calculated using a random effects or fixed effects. Heterogeneity of included studies was assessed using the I 2 test. Publication bias was examined by the Begg's funnel plot.

Results: Fifteen RCTs totaling 2,250 patients were included, compared with surgery alone (SA), the HR (95\% CI) for the overall survival (OS) was 0.88 (0.81-0.97), the disease-free survival (DFS) rate was 0.78 (0.64-0.96), and the progression-free survival (PFS) rate was $0.72(0.56-0.91)$. To clarify the effect of histological differences on CRT, a subgroup analysis was performed, and the results showed that there was no statistically significant difference in the HR of the OS in squamous cell carcinoma (SCC) and adenocarcinoma (AC) for those treated with preoperative CRT compared with SA $[\mathrm{HR}$ of SCC $=0.92$ (0.83-1.01), $\mathrm{P}=0.085$; HR of $\mathrm{AC}=0.77$ (0.55-1.08), $\mathrm{P}=0.135]$. There was however a statistically significant difference in the HR of the PFS in SCC and AC for those treated with preoperative CRT compared with SA $[\mathrm{HR}=0.48(0.32-0.71)$ for SCC and $=0.83(0.57-1.21)$ for the AC]. The indirect compare results show there was no statistically significant difference between SCC and AC in OS, ROR (=1.19, 95\% CI: 0.84-1.70), but have a statistically significant difference in PFS, ROR (=0.58, 95\% CI: 0.33-1.00).

Conclusions: Preoperative CRT can improve the OS, PFS and DFS of esophageal cancer. The results of subgroup analyses show that preoperative CRT can improve the PFS of esophageal SCC, but not esophageal AC. However, there was no significant difference in the OS of SCC and AC. And the indirect comparison results confirmed it. 
Keywords: Chemoradiation (CRT); esophageal cancer; systematic review; meta-analysis

Submitted Jul 03, 2018. Accepted for publication Oct 24, 2018.

doi: $10.21037 /$ tcr.2018.11.04

View this article at: http://dx.doi.org/10.21037/tcr.2018.11.04

\section{Introduction}

Esophageal cancer (EC) is the eighth most serious malignancy that affects human health (1), and the majority of first time patients are diagnosed with advanced esophageal carcinoma. One investigation into this issue discovered that 455,800 new esophageal cancer cases and 400,200 deaths occurred in 2012 worldwide, while the incidence of esophageal cancer varied internationally by more than 21-fold (2); eastern Asia, and eastern and southern Africa have the highest ratio and West Africa has the lowest ratio. Although treatment is improving, the prognosis of esophageal cancer is still poor, and the 5 -year overall survival (OS) rate is about $15-34 \%(3,4)$. Preoperative chemoradiation (CRT) is still the most popular treatment model $(5,6)$. Squamous cell carcinoma (SCC) and adenocarcinoma (AC) are the two main histological subtypes of EC (7). It has become clear that there are geographic variations in the histological subtypes of EC, with esophageal AC being more prevalent in many of the more developed countries, and esophageal SCC predominating in eastern Asia and Africa $(8,9)$.

There are many randomized controlled trials (RCTs) and meta-analyses of preoperative CRT for esophageal cancer; however, the clinical efficacy of preoperative CRT is still controversial. Some studies have demonstrated that the effect of preoperative CRT is superior to surgery alone (SA) $(10,11)$, but others have shown that there was no significant difference in the efficacy between preoperative CRT and SA (12).

In this study, we have collected more RCTs to clarify the OS of preoperative CRT versus SA, while using progression-free survival (PFS) and diseasefree survival (DFS) to measure the quality of patients' life with esophageal cancer. Meanwhile, we have also compared the effectiveness and safety between different histology types of esophageal cancer, and used indirect comparison methods to compare SCC and AC in preoperative CRT.

\section{Methods}

\section{Search strategy}

RCTs with preoperative CRT for esophageal cancer published in PubMed, Cochrane Library, Embase and Web of Science between January 1990 and December 2017 were identified through a systematic search. The search strategy of PubMed was as follows in Figure S1.

Supplementary search strategy of PubMed was as follows in Figure S2.

\section{Study selection}

The following criteria were used for study inclusion: (I) RCTs that compared preoperative CRT with SA; (II) no previous treatment; (III) esophageal or gastro-esophageal junction cancer (no matter what kind of histology); (IV) for studies with overlapping patients, the most recent or completed study was chosen. The exclusion criteria were as follows: (I) papers without any relevant outcomes that could be extracted for analysis; (II) papers that were not published in English or Chinese; and (III) case reports, abstracts, conference reports, reviews and reports of experiments.

\section{Identification studies}

First, two independent reviewers (WJ Ma and QN Zhang) read the title and abstract to determine which articles needed to be read in full. Second, the full text was downloaded. Finally, the articled was checked for inclusion according to the inclusion criteria described above. If reviewers could not reach consensus about an article, it was discussed with or decided by a third reader (XH Wang).

\section{Data extraction}

For each included study, the following information was extracted using a standard form: first author, year of 
publication, country, chemotherapy schedule, radiotherapy schedule, sequence, pathology, and the total sample of the experimental group and the control group.

\section{Outcome measures}

The primary outcome was the hazard ratio (HR) and $95 \%$ confidence interval ( $95 \%$ CI) of OS. Some data were calculated from the data given in the original research. Secondary outcomes were PFS and DFS.

\section{Statistical analyses}

OS, PFS and DFS were measured with HR and their corresponding $95 \%$ CI. Statistical analysis was carried out using the HR as the summary statistic. By the different histological classifications of esophageal cancer, the OS, PFS can be analyzed by sub-group. Statistical analysis was carried out using the HR as the summary statistic. STATA Version 10.0 software was used to perform all analyses. The statistical significance of the summary HR was determined with a $\mathrm{Z}$-test, and a $\mathrm{P}<0.05$ was considered as the significant threshold.

\section{Evaluation of heterogeneity and publication bias}

The heterogeneity assumption was evaluated with a chisquare-based Q-test. A P value $\geq 0.10$ for the Q-test indicated a lack of heterogeneity among studies and thus a fixed-effect model was then used, otherwise a randomeffect model was performed. Begg's and Egger's test were performed to explore publication bias when applicable (the number of included studies no less than 10 for 1 outcome).

\section{Results}

\section{Search results and trial characteristics}

A total of 1,450 published studies were identified. Among them, 16 systematic reviews were adopted, and finally, 15 studies, published from 1994 to 2015, comprising 2,250 patients, were selected for the primary analysis (Figure 1). Nine studies are about SCC alone, one RCT is about AC, and the remaining 6 studies include both patients with esophageal AC and SCC (Table 1).

\section{Survival outcomes}

\section{OS}

Ten studies include the HR and $95 \% \mathrm{CI}$; the OS of HR was 0.88 (95\% CI: $0.81-0.97, \mathrm{P}=0.007$, Figure 2), and there was a statistically significant difference between the HR for those treated with preoperative CRT compared with SA. There was a statistical heterogeneity among the studies $\left(\mathrm{I}^{2}=56.3 \%, \mathrm{P}=0.014\right)$, and a random-effect model was subsequently selected.

\section{OS based on histology}

Seven studies reported the OS of SCC and AC. The HR for OS of SCC was 0.92 (95\% CI: 0.83-1.01, P=0.085, Figure 3), and there was no significant difference in the HR of the OS of SCC for those treated with preoperative CRT and SA. The HR for OS of AC was 0.77 (95\% CI: $0.55-1.08, \mathrm{P}=0.135$, Figure 3), and there was no significant difference in the HR of the OS of SCC for those treated with preoperative CRT and SA.

\section{PFS}

Two studies reported the HR of PFS, with the overall HR of $0.71(0.59-0.86, \mathrm{P}=0.007)$, and a statistically significant difference in the HR between those treated with preoperative CRT and SA (Figure 4). There was either no or low statistical heterogeneity among the studies $\left(\mathrm{I}^{2}=35.1 \%\right.$, $\mathrm{P}=0.215)$. A fixed-effect model was subsequently selected.

\section{PFS outcomes based on SCC and AC}

In order to find out the possible factors that can affect patients with esophageal cancer, we also conducted subgroup analyses to evaluate whether or not patients with SCC or AC had different treatment outcomes following a CRT or SA. The subgroup analyses showed that the histological type of esophagus had an impact on PFS for CRT relative to SA. The HR of PFS was $0.48(0.32-0.71$, $\mathrm{P}=0.000)$ for SCC, and $0.83(0.57-1.21, \mathrm{P}=0.334)$ for the $\mathrm{AC}$ (Figure 5). The results also show that there was no or low statistical heterogeneity among the studies (SCC: $\mathrm{I}^{2}=0$, $\mathrm{P}=0.960$; AC: $\mathrm{I}^{2}=65.7 \%, \mathrm{P}=0.088$ ).

\section{Indirect comparison method to compare SCC and AC}

We used the indirect comparison methods to calculate the RHR of SCC/AC, then used the STATA Version 10.0 software to draw the plot (Figure 6), the results show that 


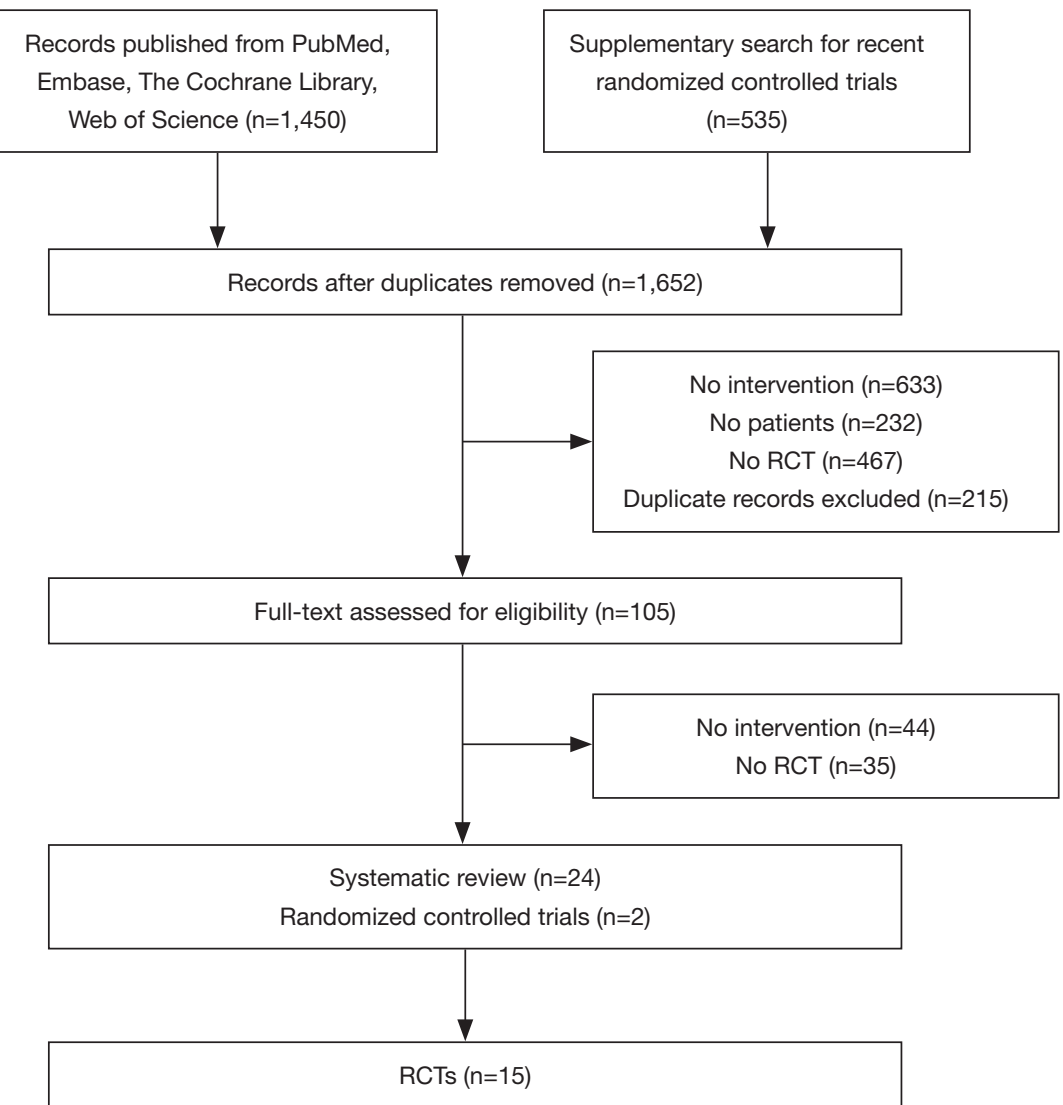

Figure 1 Diagram displaying the search and selection process of eligible trials.

there was no statistically significant difference between SCC and $\mathrm{AC}$ in OS, ROR ( $=1.19,95 \%$ CI: 0.84-1.70), but have a statistically significant difference in PFS, ROR $(=0.58,95 \%$ CI: 0.33-1.00).

\section{DFS}

Two studies reported the HR of DFS. The overall HR of DFS was $0.78(0.64-0.96, \mathrm{P}=0.018)$, and there was a statistically significant difference in the HR of DFS between those treated with preoperative CRT compared with SA (Figure 7). The results also show that there was no or low statistical heterogeneity among the studies $\left(\mathrm{I}^{2}=29.8 \%\right.$, $\mathrm{P}=0.233)$.

\section{Publication bias}

We used the HR of OS to test the publication bias and found evidence for publication bias. The $\mathrm{P}$ value based on Egger's test for OS of 0.016 , and the $\mathrm{P}$ value based on the Begg's test for OS of 1 , also indicates the existence of publication bias.

\section{Discussion}

Esophageal cancer is an aggressive disease with poor prognosis, and is currently treated with CRT after surgery as a standard procedure (29). In this meta-analysis, we used HR and $95 \%$ CI to measure the survival outcomes. We observed that preoperative CRT could improve the OS, PFS and DFS compared with SA. We also conducted subgroup analyses of the histological types of esophageal cancer to reduce the heterogeneity of the article. The subgroup results show that there was no significant difference between preoperative CRT and SA in the OS for esophageal SCC and AC. However, with the subgroup of PFS results indicating that preoperative CRT is more effective for esophageal SCC. And the indirect compare results also confirmed it. Finally, we conducted a metaanalysis of DFS, with the results also showing that the preoperative CRT could improve the DFS. 


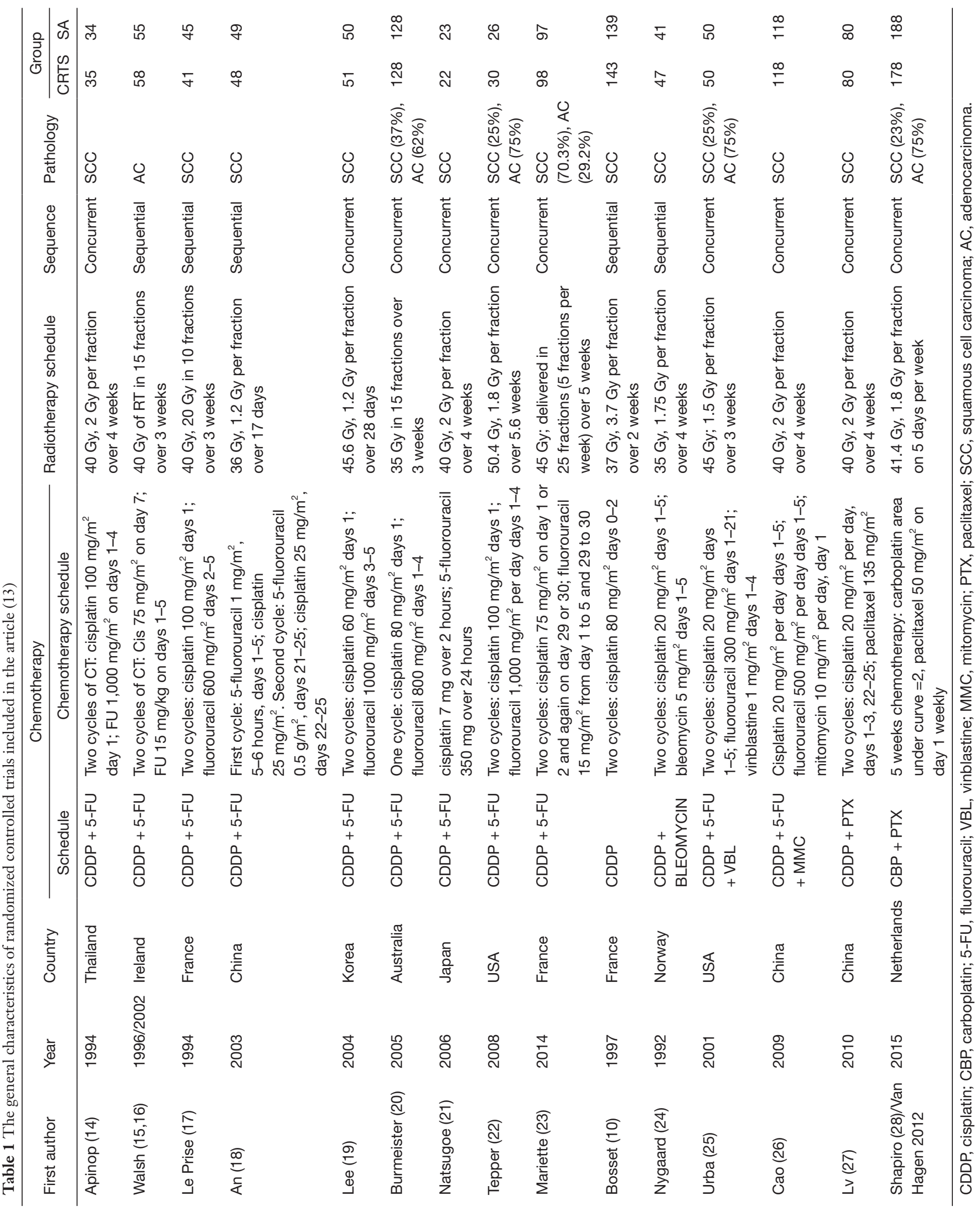




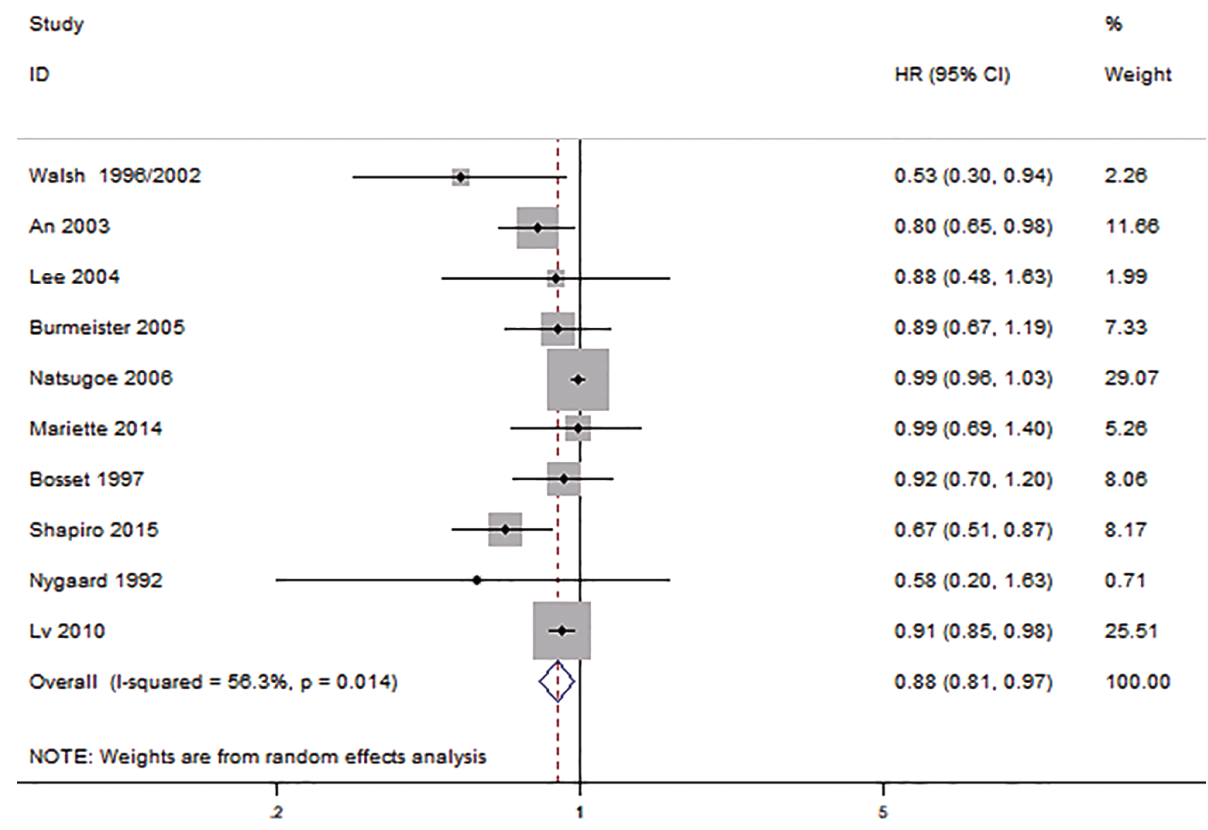

Figure 2 Forest plot of overall survival outcomes (CRTS vs. SA). Hazard ratios (HR) are shown with 95 percent confidence intervals (95\% CIs). Weights (represented by the size of square plotted for each study) are from random-effect analysis. $\mathrm{P}=0.007$.

Study

ID

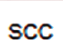

Shapiro 2015

Burmeister 2005

Bosset 1997

Lee 2004

Natsugoe 2008

Lv 2010

Subtotal (I-squared $=81.0 \%, p=0.025$ )

.

AC

Shapiro 2015

Burmeister 2005

Walsh 1996/2002

Subtotal (I-squared $=58.3 \%, p=0.101$ )

Overall (I-squared $=61.7 \%, p=0.007$ )

NOTE: Weights are from random effects analysis

28

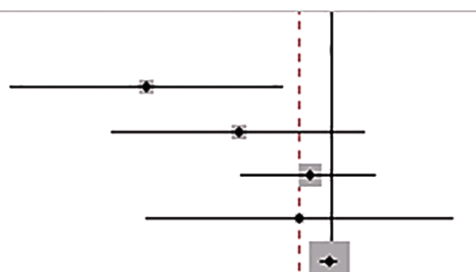

HR $(95 \% \mathrm{Cl})$

$\%$

Weight

$0.48(0.28,0.83) \quad 3.30$

$0.69(0.42 .1 .15) \quad 3.78$

$0.92(0.70,1.20) \quad 10.27$

$0.88(0.48 .1 .63) \quad 2.66$

$0.99(0.98,1.03) \quad 31.91$

$0.91(0.85 .0 .98) \quad 28.67$

$0.92(0.83,1.01) \quad 80.59$

$0.73(0.55 .0 .98) \quad 9.32$

$1.04(0.74,1.48) \quad 7.08$

$0.53(0.30 .0 .94) \quad 3.01$

$0.77(0.55,1.08) \quad 19.41$

$0.88(0.79,0.98) \quad 100.00$

Figure 3 Forest plot of overall survival outcomes (CRTS vs. SA) based on SCC and AC. Data are pooled using a random effects model. SCC: $\mathrm{P}=0.085$, AC: $\mathrm{P}=0.135$. SCC, squamous cell carcinoma; AC, adenocarcinoma. 


\section{Study}

D

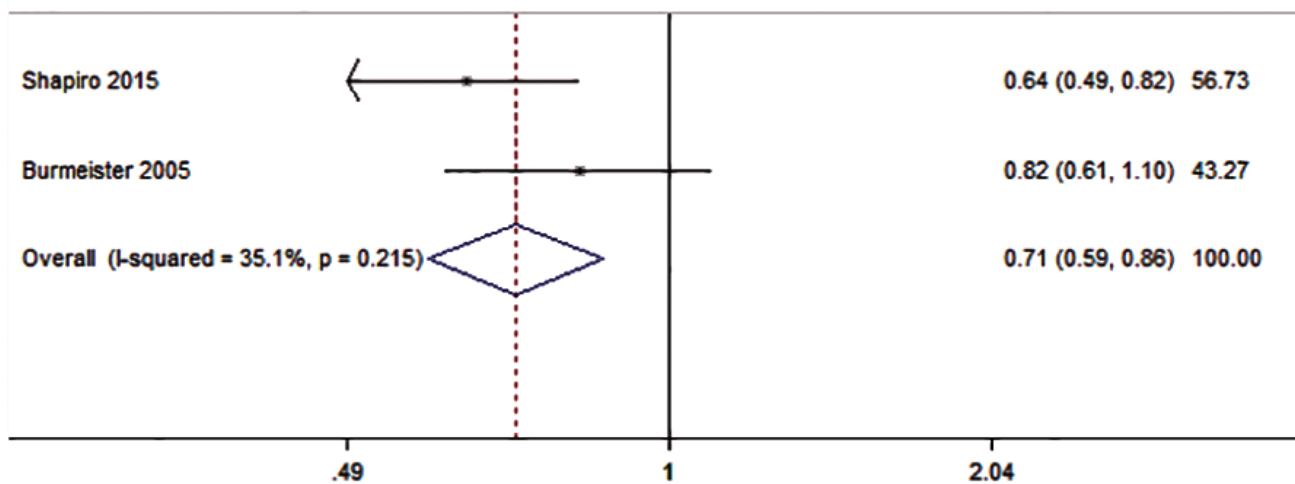

Figure 4 Forest plot of progression-free survival outcomes (CRTS vs. SA). Data are pooled using a fixed effects model. The P value was 0.007.

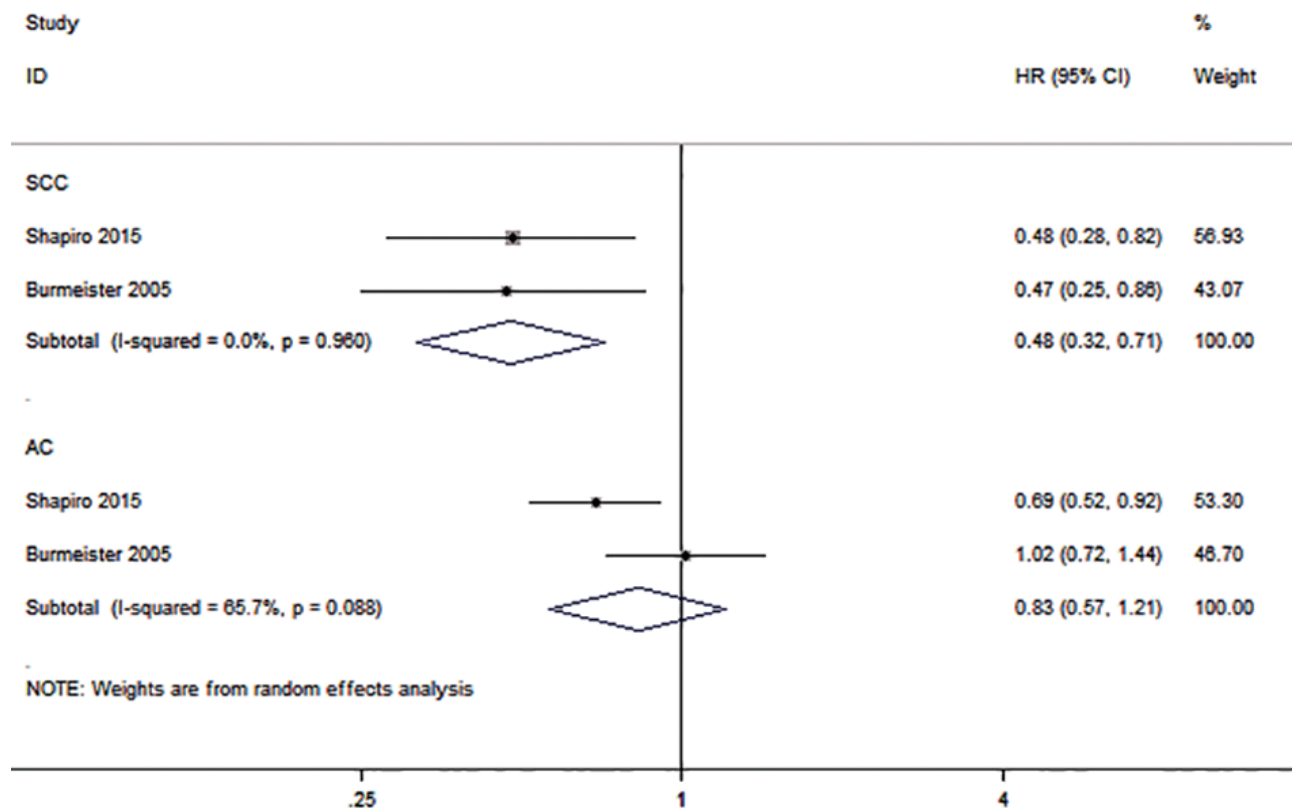

Figure 5 Forest plot of progression-free survival outcomes (CRTS vs. SA) based on SCC and AC. Data are pooled using a random effects model. The P value of SCC was 0.000, AC was 0.334. SCC, squamous cell carcinoma; AC, adenocarcinoma.

The treatment of preoperative CRT for esophageal cancer is still controversial. Greer et al. and other researchers believe that the preoperative CRT and SA show no difference in the efficacy of esophageal cancer's treatment $(30,31)$. On the other hand, Sjoquist et al. proposed that the effect of preoperative CRT was better than SA for esophageal cancer $(3,32)$. We have screened
15 RCTs from different databases appearing between 1994 and 2015. Compared with previous meta-analyses, our study has a large sample size, a wider range of studies, and a higher degree of confidence. This article complements the previously included meta-analyses articles with new RCTs. Among these studies, van Hagen et al.'s (33) and Shapiro et al.'s (28) research designs were identical, but their follow- 


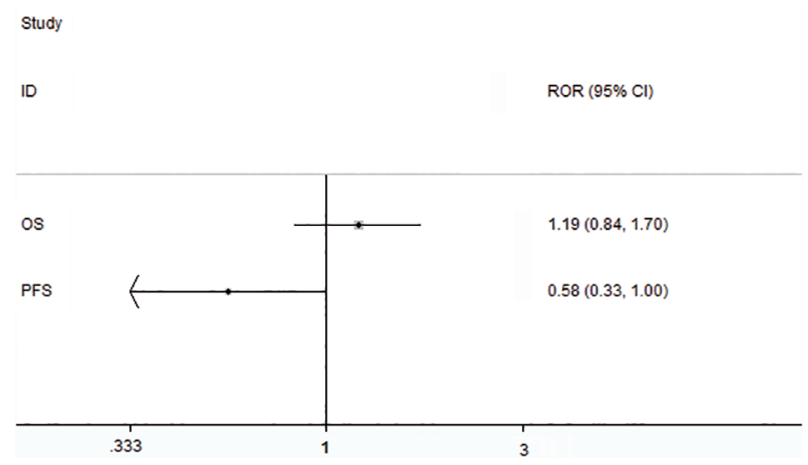

Figure 6 The plot of indirect comparison method to compare SCC and AC of OS and PFS. SCC, squamous cell carcinoma; AC, adenocarcinoma; OS, overall survival; PFS, progression-free survival.

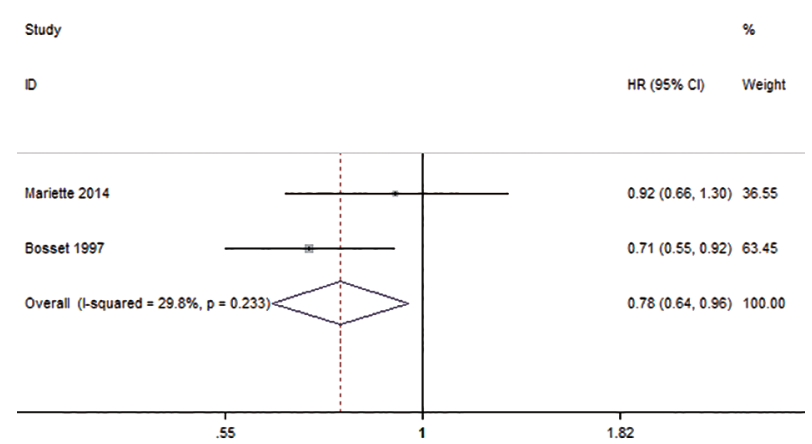

Figure 7 Forest plot of disease-free survival outcomes (CRTS vs. $\mathrm{SA})$ using a random effects model. The $\mathrm{P}$ value was 0.018 .

up time and outcome measures were different. The reason why our article included the Shapiro study (28) was because not only was it recently published, but also because it contained the outcome index PFS that we needed. The van Hagen article (33), however, only contained the OS.

Our meta-analysis shows that the results of preoperative CRT for OS, and PFS and DFS, were all better than SA with esophageal cancer. Meanwhile, the subgroup analyses showed that there was no significant difference between SCC and AC in OS, but the PFS subgroup did demonstrate that the SCC had a better effect compared with AC. The results also showed that there was some heterogeneity in the groups. We performed a sensitivity analysis and subgroup analysis but did not reduce the heterogeneity. Possible sources of these heterogeneities might have originated from the fact that Shapiro's studies included both esophageal cancer and cancer at the junction, while Walsh et al.'s (15) and Burmeister et al.'s (20) studies included only patients with esophageal cancer. Second, different doses of chemotherapy and radiotherapy in addition to different treatment time might have contributed to the heterogeneity. Finally, the orders of CRT were different: Walsh used sequential CRT, while Shapiro et al. (28) and Burmeister et al. (20) both used concurrent CRT.

Compared with SA, a preoperative CRT significantly improved the PFS for patients with esophageal SCC. However, there was no clear effect on patients with esophageal AC. The RCTs included in this paper are also divided into two different groups. One group considered preoperative CRT to be clinically relevant to both SCC and AC subtypes, while the other group considered preoperative CRT to have a significant correlation with SCC, but no significant correlation to AC. This may be related to the difference in chemotherapy cycles, radiotherapy doses, and tumor location of patients in these two trial types.

The efficacy of the preoperative CRT on SCC status has been proven to be very effective in many meta-analyses, yet the efficacy of preoperative CRT on AC is controversial. Although RCTs have demonstrated that preoperative CRT is effective for both SCC and AC, no meta-analysis has yet been performed to confirm this conclusion. There were two meta-analyses $(34,35)$ showing that preoperative CRT can improve OS, and our own meta-analysis confirmed this finding. Despite this, in our meta-analyses we also found that there was no difference between preoperative CRT and SA compared with SCC and AC of OS, while the PFS for SCC and AC showed that the CRTS for SCC had a better efficiency than AC. Therefore, as the incidence of AC and SCC of esophageal cancer increases around the world each year, there is an urgent need for future scientific studies to find effective ways to improve the survival rate of $\mathrm{AC}$ and SCC patients respectively.

Our study has some limitations. First, although 4 medical English-language databases were searched, inevitably there were relevant studies that might have been missed. Second, this article only includes articles in Chinese and English, which greatly limits the number of included trials.

For patients who are suffering from cancer, the aim of healthcare interventions is to cure or considerably prolong life and to ensure the best possible quality of life for cancer survivors (36). There are already many studies proving that the current standard treatment of preoperative CRT for esophageal cancer conveys far more benefits for esophageal SCC than for esophageal AC. Therefore, as the study of patients with esophageal cancer continues to deepen, the search for suitable therapeutic methods for both esophageal 
SCC and AC is the direction we need to continue to explore.

\section{Conclusions}

The results of this study show that a preoperative CRT scan can improve the OS, PFS and DFS in patients with esophageal cancer when compared with just providing a surgery treatment alone. Additionally, the subgroup of PFS results show that compared with esophageal AC, esophageal SCC can benefit from preoperative CRT. On the other hand, the subgroup of OS results shows that there was no difference between esophageal SCC and AC. More randomized controlled studies are needed to determine whether preoperative CRT for the treatment of esophageal SCC and AC really has a significant difference. Therefore, the future direction of clinicians is to try to find the most suitable treatments for both esophageal SCC and AC.

\section{Acknowledgments}

The authors would like to thank Kehu Yang (Evidence Based Medicine Center of Lanzhou University) for the support given to the methodology.

Funding: Clinical Application Specification and Verification of Esophageal Cancer Based on Domestic Precision Radiotherapy Equipment (Approval No. 2016YFC0105706).

\section{Footnote}

Conflicts of Interest: All authors have completed the ICMJE uniform disclosure form (available at http://dx.doi. org/10.21037/tcr.2018.11.04). The authors have no conflicts of interest to declare.

Ethical Statement: The authors are accountable for all aspects of the work in ensuring that questions related to the accuracy or integrity of any part of the work are appropriately investigated and resolved.

Open Access Statement: This is an Open Access article distributed in accordance with the Creative Commons Attribution-NonCommercial-NoDerivs 4.0 International License (CC BY-NC-ND 4.0), which permits the noncommercial replication and distribution of the article with the strict proviso that no changes or edits are made and the original work is properly cited (including links to both the formal publication through the relevant DOI and the license). See: https://creativecommons.org/licenses/by-nc-nd/4.0/.

\section{References}

1. Pennathur A, Gibson MK, Jobe BA, et al. Oesophageal carcinoma. Lancet 2013;381:400-12.

2. Torre LA, Bray F, Siegel RL, et al. Global cancer statistics, 2012. CA Cancer J Clin 2015;65:87-108.

3. Sjoquist KM, Burmeister BH, Smithers BM, et al. Survival after neoadjuvant chemotherapy or chemoradiotherapy for resectable oesophageal carcinoma: an updated metaanalysis. Lancet Oncol 2011;12:681-92.

4. Fakhrian K, Ordu AD, Lordick F, et al. Long-term outcomes of trimodality treatment for squamous cell carcinoma of the esophagus with cisplatin and/or 5-FU: more than 20 years' experience at a single institution. Strahlenther Onkol 2014;190:1133-40.

5. Fan M, Lin Y, Pan J, et al. Survival after neoadjuvant chemotherapy versus neoadjuvant chemoradiotherapy for resectable esophageal carcinoma: A meta-analysis. Thorac Cancer 2016;7:173-81.

6. Wijnhoven BP, van Lanschot JJ, Tilanus HW, et al. Neoadjuvant chemoradiotherapy for esophageal cancer: a review of metaanalyses. World J Surg 2009;33:2606-14.

7. Malhotra GK, Yanala U, Ravipati A, et al. Global trends in esophageal cancer. J Surg Oncol 2017;115:564-79.

8. Rustgi AK, El-Serag HB. Esophageal carcinoma. N Engl J Med 2014;371:2499-509.

9. Arnold M, Soerjomataram I, Ferlay J, et al. Global incidence of oesophageal cancer by histological subtype in 2012. Gut 2015;64:381-7.

10. Bosset JF, Gignoux M, Triboulet JP, et al. Chemoradiotherapy followed by surgery compared with surgery alone in squamouscell cancer of the esophagus. N Engl J Med 1997;337:161-7.

11. Fiorica F, Di Bona D, Schepis F, et al. Preoperative chemoradiotherapy for oesophageal cancer: a systematic review and meta-analysis. Gut 2004;53:925-30.

12. Bass GA, Furlong H, O'Sullivan KE, et al. Chemoradiotherapy, with adjuvant surgery for local control, confers a durable survival advantage in adenocarcinoma and squamous cell carcinoma of the oesophagus. Eur J Cancer 2014;50:1065-75.

13. Wang F, Wang YM, He W, et al. Chemoradiotherapy followed by surgery could improve the efficacy of treatments in patients with resectable esophageal carcinoma. Chin Med J (Engl) 2013;126:3138-45.

14. Apinop C, Puttisak P, Preecha N. A prospective study of combined therapy in esophageal cancer. Hepatogastroenterology 1994;41:391-3.

15. Walsh TN, Noonan N, Hollywood D, et al. A comparison 
of multimodal therapy and surgery for esophageal adenocarcinoma. N Engl J Med 1996;335:462-7.

16. Walsh TN, McDonell CO, Mulligan ED, et al. Multimodal therapy versus surgery alone for squamous cell carcinoma of the esophagus: a prospective randomized trial. Gastroenterology 2000;118:1008.

17. Le Prise E, Etienne PL, Meunier B, et al. A randomized study of chemotherapy, radiation therapy, and surgery versus surgery for localized squamous cell carcinoma of the esophagus. Cancer 1994;73:1779-84.

18. An FS, Huang JQ, Xie YT, et al. A prospective study of combined chemoradiotherapy followed by surgery in the treatment of esophageal carcinoma. Zhonghua Zhong Liu Za Zhi 2003;25:376-9.

19. Lee JL, Park SI, Kim SB, et al. A single institutional phase III trial of preoperative chemotherapy with hyperfractionation radiotherapy plus surgery versus surgery alone for resectable esophageal squamous cell carcinoma. Ann Oncol 2004;15:947-54.

20. Burmeister BH, Smithers BM, Gebski V, et al. Surgery alone versus chemoradiotherapy followed by surgery for resectable cancer of the oesophagus: a randomised controlled phase III trial. Lancet Oncol 2005;6:659-68.

21. Natsugoe S, Okumura H, Matsumoto M, et al. Randomized controlled study on preoperative chemoradiotherapy followed by surgery versus surgery alone for esophageal squamous cell cancer in a single institution. Dis Esophagus 2006;19:468-72.

22. Tepper J, Krasna MJ, Niedzwiecki D, et al. Phase III trial of trimodality therapy with cisplatin, fluorouracil, radiotherapy, and surgery compared with surgery alone for esophageal cancer: CALGB 9781. J Clin Oncol 2008;26:1086-92.

23. Mariette C, Dahan L, Mornex F, et al. Surgery alone versus chemoradiotherapy followed by surgery for stage I and II esophageal cancer: Final analysis of randomized controlled phase III trial FFCD 9901. J Clin Oncol 2014;32:2416-22.

24. Nygaard K, Hagen S, Hansen HS, et al. Pre-operative radiotherapy prolongs survival in operable esophageal carcinoma: a randomized, multicenter study of preoperative radiotherapy and chemotherapy. The second Scandinavian trial in esophageal cancer. World J Surg 1992;16:1104-9.

25. Urba SG, Orringer MB, Turrisi A, et al. Randomized trial of preoperative chemoradiation versus surgery alone in patients with locoregional esophageal carcinoma. J Clin Oncol 2001;19:305-13.

26. Cao XF, He XT, Ji L, et al. Effects of neoadjuvant radiochemotherapy on pathological staging and prognosis for locally advanced esophageal squamous cell carcinoma. Dis Esophagus 2009;22:477-81.

27. Lv J, Cao XF, Zhu B, et al. Long-term efficacy of perioperative chemoradiotherapy on esophageal squamous cell carcinoma. World J Gastroenterol 2010;16:1649-54.

28. Shapiro J, van Lanschot JJB, Hulshof MCCM, et al. Neoadjuvant chemoradiotherapy plus surgery versus surgery alone for oesophageal or junctional cancer (CROSS): long-term results of a randomised controlled trial. Lancet Oncol 2015;16:1090.

29. Deng HY, Wang WP, Wang YC, et al. Neoadjuvant chemoradiotherapy or chemotherapy? A comprehensive systematic review and meta-analysis of the options for neoadjuvant therapy for treating oesophageal cancer. Eur J Cardiothorac Surg 2017;51:421.

30. Greer SE, Goodney PP, Sutton JE, et al. Neoadjuvant chemoradiotherapy for esophageal carcinoma: a metaanalysis. Surgery 2005;137:172-7.

31. Urschel JD, Vasan H. A meta-analysis of randomized controlled trials that compared neoadjuvant chemoradiation and surgery to surgery alone for resectable esophageal cancer. Am J Surg 2003;185:538-43.

32. Wang DB, Zhang X, Han HL, et al. Neoadjuvant chemoradiotherapy could improve survival outcomes for esophageal carcinoma: a meta-analysis. Dig Dis Sci 2012;57:3226-33.

33. van Hagen P, Hulshof MC, van Lanschot JJ, et al. Preoperative chemoradiotherapy for esophageal or junctional cancer. N Engl J Med 2012;366:2074-84.

34. Jin HL, Zhu H, Ling TS, et al. Neoadjuvant chemoradiotherapy for resectable esophageal carcinoma:A meta-analysis. World J Gastroenterol 2009;15:5983-91.

35. Fu T, Bu ZD, Li ZY, et al. Neoadjuvant chemoradiation therapy for resectable esophago-gastric adenocarcinoma: a meta-analysis of randomized clinical trials. BMC Cancer 2015;15:322.

36. Ge L, Tian JH, Li XX, et al. Epidemiology Characteristics, Methodological Assessment and Reporting of Statistical Analysis of Network Meta-Analyses in the Field of Cancer. Sci Rep 2016;6:37208.

Cite this article as: Ma WJ, Zhang QN, Shi SZ, Zhang XY, Zhao Y, Tian JH, Wang XH. Preoperative chemoradiation may be more effective for esophageal squamous cell carcinoma compared with adenocarcinoma: results from 15 randomized controlled trials of 2,250 patients. Transl Cancer Res 2018;7(6):1421-1430. doi: 10.21037/tcr.2018.11.04 
\#1 "esophageal Neoplasms"[Mesh] OR esophagus cancer[Title/Abstract] OR esophagus tumour[Title/Abstract] OR esophagus tumor[Title/Abstract] OR esophagus neoplasms[Title/Abstract] OR oesophagus cancer[Title/Abstract] OR oesophagus tumour[Title/Abstract] OR oesophagus tumor[Title/Abstract] OR oesophagus neoplasms[Title/Abstract] OR esophageal cancer[Title/Abstract] OR esophageal tumour[Title/Abstract] OR esophageal tumor[Title/Abstract] OR esophageal neoplasms[Title/Abstract] OR oesophageal cancer[Title/Abstract] OR oesophageal tumour[Title/Abstract] OR oesophageal tumor[Title/Abstract] OR oesophageal neoplasms[Title/Abstract]

\#2 "Radiotherapy"[Mesh] OR "Chemoradiotherapy"[Mesh] OR "Brachytherapy"[Mesh] radiotherapy[Title/ Abstract] OR Radiotherapies[Title/Abstract] OR radiation[Title/Abstract] OR Brachytherapy[Title/Abstract] OR Chemoradiotherapy[Title/Abstract]

\#3 "Meta-Analysis" [Publication Type] OR "Meta-Analysis as Topic"[Mesh] OR meta analysis[Title/Abstract] OR meta analyses[Title/Abstract] OR meta-analysis[Title/Abstract] OR meta-analyses[Title/Abstract] OR systematic review[Title/ Abstract] OR systematic reviews[Title/Abstract] OR metaanalysis[Title/Abstract] OR metaanalyses[Title/Abstract] \#4 \#1 AND \#2 AND \#3

Figure S1 The search strategy of PubMed.

\#1 "esophageal Neoplasms"[Mesh] OR esophagus cancer[Title/Abstract] OR esophagus tumour[Title/Abstract] OR esophagus tumor[Title/Abstract] OR esophagus neoplasms[Title/Abstract] OR oesophagus cancer[Title/Abstract] OR oesophagus tumour[Title/Abstract] OR oesophagus tumor[Title/Abstract] OR oesophagus neoplasms[Title/Abstract] OR esophageal cancer[Title/Abstract] OR esophageal tumour[Title/Abstract] OR esophageal tumor[Title/Abstract] OR esophageal neoplasms[Title/Abstract] OR oesophageal cancer[Title/Abstract] OR oesophageal tumour[Title/Abstract] OR oesophageal tumor[Title/Abstract] OR oesophageal neoplasms[Title/Abstract]

\#2 "Radiotherapy"[Mesh] OR "Chemoradiotherapy"[Mesh] OR "Brachytherapy"[Mesh] radiotherapy[Title/ Abstract] OR Radiotherapies[Title/Abstract] OR radiation[Title/Abstract] OR Brachytherapy[Title/Abstract] OR Chemoradiotherapy[Title/Abstract]

\#3 "Clinical Trials, Phase II as Topic"[Mesh] OR "Clinical Trials, Phase III as Topic"[Mesh] OR "Clinical Trials, Phase IV as Topic"[Mesh] OR "Controlled Clinical Trials as Topic"[Mesh] OR "Randomized Controlled Trials as Topic"[Mesh] OR "Intention to Treat Analysis"[Mesh] OR "Pragmatic Clinical Trials as Topic"[Mesh] OR "Clinical Trials, Phase II"[Publication Type] OR "Clinical Trials, Phase III"[Publication Type] OR "Clinical Trials, Phase IV"[Publication Type] OR "Controlled Clinical Trials"[Publication Type] OR "Randomized Controlled Trials"[Publication Type] OR "Pragmatic Clinical Trials as Topic"[Publication Type] OR "Single-Blind Method"[Mesh] OR "Double-Blind Method"[Mesh] OR random*Title/Abstract] OR blind*[Title/Abstract] OR singleblind*[Title/Abstract] OR doubleblind*[Title/Abstract] OR tripleblind*[Title/Abstract] \#4 \#1 AND \#2 AND \#3

Figure S2 Supplementary search strategy of PubMed. 